smancipeted is to form new individuals by agamogenesis. But in the higher animals this never happens, because the emaricipation is always more or less incomplete; hence in them only new parts, new tissues, and neoplasms are formed. Here the nature of the product seems to depend upon the grade of organisation attained by the neoplastic cells. It is well known that in the formation of highly organised structures the cells of the part suffer loss or impairment of their proliferous (reproductive) power, owing to their protoplasm being used up and converted into special tissues. Most non-malignant neoplasms are of this nature; their histological character closely resembles that of the corresponding normal tissue, whence they originate. Such neoplasmis lack infective properties because they are highly organised. On the other hand, all lowly organised neoplasms are more or less malignant, and the most malignant are the most lowly organised. Hence it may be concluded that the degree of malignancy of a given neoplasm is chiefly dependent upon its grade of organisation. To this cause, and not to any essential difference in nature of the morbid process, I attribute the different behaviour of these two kinds of neoplasms.

Viewing the matter in this light, I can see no probability of there being any truth in the theory that neoplasms are the outcome of general blood disease, dependent upon the presence of micro-organisms like tubercle and syphilis. I think De Morgan came nearer the truth than anyone else when, in the memorable debate on cancer at the Pathological Society in 1874, he said, "I can see no analogy between new growth, whether as innocent as lipoma or ss malignant as cancer, and the products of true general or blood disease in respect to their genesis and destination. From the first a tumour is a living, self-dependent formation, capable of continual growth by virtue of its own power of using the nutritive materials supplied to it. Nothing like this is seen in any of the blood diseases."

\section{THE TREATMENT OF ACUTE RHEUMATISM, WITH SPECIAL REFERENCE TO THE USE OF THE SALICYLATES, ${ }^{1}$}

By DONALD W. C. HOOD, M.D. CanTaB., PHYSICIAN TO THE WEST LONDON HOSPITAL.

THIs paper is based upon 2200 cases of acute sthenic rheumatism occurring in both sexes under thirty-six years of age. Of these cases, 850 were taken from the clinical records of Guy's Hospital before the introduction of salicylates; the remaining cases were all treated with alicylates, the remedy being in almost every instance salicylate of soda; the dose uniform-twenty grains, every one, two, or more hours. Through the great courtesy of the staff of St. Bartholomew's Hospital, 515 of these cases have been drawn from the clinical reports of that hospital; the remaining cases, without exception, are from Guy's.

The introduction of the salicylates has completely revolutionised the treatment of acute rheumatism, and the time appears now ripe to inquire into the advantages of this specific treatment, and it appears right to ask if with these sdvantages there may not, at least in some cases, be corresponding disadvantages which a mor $\theta$ complete and exact knowledge of the action of the drug may prevent or mitigate. An analysis of this large number of cases was conducted principally for the purpose of estimating the comparative results in the case of patients treated specifically, and on general principles, (1) with regard to the suffering due to the joint effusion, (2) the length of illness, (3) the percentage of cases affected with cardiac complications, (4) the mortality, and (5) the dangerous symptoms which many observers state follow the use of salicylates.

With regard to the pain due to joint effusion there cannot be any doubt that patients treated with salicylates in many cases experience a marked and rapid diminution or tota cessation of pain, and parallel with this loss of pain there is frequently an $\epsilon$ qually rapid fall of temperature. Tables were prepared showing that in the case of 728 patients treated with salicylates, 582 lost their pains within seven days.

1 Abstract of paper read before the Medical Society of London, Feb. 13th, 1888 .
Making full allowance for the heavy list of relapses-namely 137,- - these figures d warf those derived from patients treated on general principles, for here wo find that, among 612, 140 only lost their pain in a similar period of time. Complete relief from pain within three days was noted in 300 patients treated at St. Bartholomew's out of a total of 515. Forty per cent. of these relapsed, the word "relapse" not being made use of unless there had been decided return of joint pain accompanied by a temperature above $100^{\circ}$, the condition lasting longer than twenty-four hours. In many cases it was noted that the illness following on a relapse was of more intense character, being marked with higher temperature than noted at the initial seizure, and, again, in most cases was complicated with cardiac mischief. Pain and stiffness of joints appeared more general af ter the fever had subsided, among patients treated specifically, than among those treated on general principles.

Although all observers do not exactly agree as to the relative frequency with which acute rheumatism is complicated with cardiac lesions, the various estimates closely coincide, and may be said to indicate from 50 to 60 per cent. of patients as likely to have cardiac complications. With this estimate the figures quoted will be found to agree. It has been impossible to differentiate between different forms of cardiac disease. Among 850 patients treated on general principles, 500 were noted as having cardiac mischief. Examining the salicylate cases in series as they were collected, of 328 cases from Gay's 190 were affected. In a second series from Guy's, of 360 cases 241 had cardiac complication and, again, in 515 cases from st. Bartholomew's 316 were affected. A study of these figures purposely reported in series shows how extremely difficult it is to form an exact numerical estimate of the comparative frequency with which the heart is affected in acute rheumatism. Taking any one of these series by itself, a different conclusion would be arrived at from that obtained by considering the cases in aggregate. If the total number of cases be taken, we find that among the salicylate series slightly over 60 per cent. were affected, whereas in the case of patients treated without salicylate slightly under 60 per cent. were affected. Practically these percentages agree, and enforce the impression gained from studying a large number of cases that the salicylates have no effect whatever in either reducing, preventing, or limiting the intensity of cardiac inflammation occurring during the course of acute rheumatism.

From the records of St. Bartholomew's ninety-five cases were taken, showing the commencement of cardiac disease after the patient was admitted into hospital, where, on admission, the heart having been carefully examined, no trace of disease had been found, and where the patient at the time of the inflammatory attack was thoroughly under the influence of salicylates. Estimating the value of the specific remedy in cases of primary rheumatism, it was found that of 516 patients treated without salicylates 290 were affected with cardiac complication; of 736 cases of primary rheumatism treated with salicylates 402 had cardiac complication; the proportion between the two groups being almost identical.

Tables were constructed to show the effect of treatment as influenced by the length of illness before admission; i.e., before the patient was placed under treatment, specific or otherwise, 1089 cases were so tabulated, 507 being treated without salicylates and 582 with that drug. The study of these cases showed little or no difference between the two groups.

Attention was drawn to the dangerous symptoms observed following the use of the drug, and Dr. Broadbent's remarks, when president of the Clinical Society, were quoted, showing the necessity of making some such inquiry. The author stated, from his own study of the matter, dangerous symptoms occurred in many cases where the temperature had fallen to normal, and the pains had completely subsided. In these cases furious delirium ushered in hyperpyrexia, with fatal results. In order to make the inquiry more complete, advantage had been taken of all published cases in THE LANCET since the year 1864. In twelve years, 1864-75 there are 19 fatal cases mentioned; in 16 of these death was attributed to hyperpyrexia. These cases all occurred prior to the introduction of salicylates. In ten years and six months-1876 to June, 1886-24 fatal cases are reported as occurring among patients treated by salicylates; in 20 of these cases the fatal result was attributed to hyperpyrexia. These published cases were not referred to for the purpose of estimating the comparative mortality, but rather with the view of drawing attention to the fact-which has been 
disputed - that patients under the influence of salicylates, having lost their pain and fever, may yet have a sudden rise of temperature amounting to hyperpyrexia, such condition being often accompanied with delirium of a furious character. The leading features of such cases were mentioned, brief details of 9 being given.

Anticipating the retort that in at least some of these cases the drug had not been used in sufficient quantity, several cases were quoted in which very large and oftrepeated doses had been used-i.e., one where fifty grains of salicin had been given every hour, and another where salicylate of soda had been given in twenty-grain doses every hour for two days, with relief of pain and fever, but where hyperpyrexia occurred while the patient was under these large doses. Again, a series of cases were alluded to two falling under the direct notice of the author, in which the alarming symptoms ceased on the drug being withdrawn. Attention was also directed to toxic effects occurring in a few cases in which only very small quantities of the remedy had been used, in these cases having produced a grave state of cardiac depression. A careful investigation into these and kindred cases proves that in salicylates we have no safeguard against hyperpyrexia, and that when a patient treated with these remedies has a rising temperature the drug should be at once discontinued, and other methods adopted for controlling this dangerous complication.

A high temperature was shown not to be incompatible with the ordinary administration of salicylates, a higher temperature than that found in the initial attack being especially noticed in the frequent relapses observed when patients are treated with salicylates. In this respect the relapses under salicylates differ from those observed among patients treated on general principles. Among 850 patients treated without salicylates, a temperature of $103^{\circ}$ was noted in 70, delirium being bat seldom alluded to, furious delirium never. Of 505 patients treated at St. Bartholomew's with salicylates 146 had a temperature of $103^{\circ}$ and upwards; 20 of these had delirium. Again, of 386 patients from Guy's, all treated with salicylates, 66 had a temperature of $103^{\circ}$ and above, 57 being noted as suffering from delirium, and in many instances the delirium was specially mentioned as being of furious character.

Attention was particularly drawn to the fact that, clinically, acute rheumatism is but rarely complicated with even the mildest form of delirium, and the passage from Senator's article was read substantiating this statement. Again, it was urged that the usual clinical course of acute rheu matism was marked by a temperature highest at the initial outbreak, which fact has been emphasised by Wunderlich. And, inasmuch as patients treated with salicylates are peculiarly prone to delirium - a symptom hitherto looked upon as of the gravest omen,-caution was called for in the use of a remedy which in many cases evidently produced a delirious condition.

The author stated that a careful study of a large number of cases could not in any way support the view that in salicylates were to be found specifics against acute rheumatism. If in truth a specific, the drug should show an overwhelming antagonistic influence, not only to the pain accompanying acute rheumatism, but also to the complications with which its path is beset. Such complications, by reason of their frequency, have come to be looked on as factors in the disease. Factors they are not. Any cardiac, pulmonary or nervous complication affecting the initial attack being due directly to the materies morbi of the disease, any remedy specific against the materies morbi must necessarily be specific against the various effects of such poison, if given in time and in sufficient quantity. Endocarditis, pericarditis, and pleurisy are as much the result of the disease as the painful swollen joint, and as such should be directly influenced by the remedy. There is, however, abundant proof that such is not the case, these complications appearing while the patient is under large and oft-repeated doses of salicylates.

In a second part of the paper the various kinds or classes of acute rheumatism were discussed, and an attempt made to formulate rules for help in their treatment. Clinically, acute rheumatism has been found to fall into one of three great groups: First, that as seen in child-life, where the tendency to cardiac complication without concomitant joint pain is so frequently met with, in which group salicylates were not considered advisable, in many such cases doing harm by producing cardiac debility. Secondly, a group embracing the rheumatism of early adult life, characterised by all the well-known symptoms of rheumatic fever - a group which, for convenience of treatment, can be further subdivided into three minor divisions: (a) acute sthenic rheumatism; (b) subacute attacks, differing from the last not only in intensity, but often in having a prolonged invasion period; and $(c)$ thirdly, the subjects of unstuble nerve power, whether such a state of nervous system be the result of a congenital peculiarity or have been induced by alcoholism. Patients falling within each of these three divisions are benefited by a different treatment, the first only being very directly helped by salicylates. In the second, salicylates may be often used as an anodyne, and may be combined with a more general and tonic treatment, while with patients whose nervous system is easily disturbed, and in whom there is tendency to delirium, salicylates should be only given with extreme caution, and are often highly dangerous.

Acute rheumatic attacks of late adult life present many anomalous characters-anomalies sufficient to warrant these cases being placed in another group. Many of these border on gout. Here there is not the same tendency to cardiac complication. Salicylates may be given as an anodyne. The drug should not be pushed; and where it has been given for forty-eight hours without reducing the temperature, notwithstanding the fact that pain has completely subsided, it should be discontinued. The remedy seems to be contraindicated in cases where relapse rapidly follows relapse. The propriety of continuing salicylates in cases where the attack has been complicated by one of the severer forms of cardiac or pulmonary inflammation was considered, the evidence being that in such cases it was advisable to discontinue their use.

\section{ADDITIONAL SERIES OF ELEVEN CASES} OF CHOLECYSTOTOMY.

\section{Bx LA W S N TA I T, F.R.C.S.,}

PROFESSOR OF GYNACOLOGY, QUHEN'S COLIRGE, BIRMINGHAMI.

IN the columns of THE LANCET of August 29th, 1885, I published the last statement of a series of operations on the gall-bladder, and these amounted to thirty cases with on death. The result in this fatal case was not attributable to the operation, but to the progress of the disease, the operation itself having no influence either one way or the other on the result of the case. Of the other thirty cases all are still living, so far as I know, with the exception of on $\theta$ who has since died of phthisis. In none of them is there any indication of a recurrence of the disease. I am now in a position to add the annexed list of eleven cases, all of which have recovered but one, and in all of the ten recoveries complete relief from the symptoms has already been obtained. (See Table.) The fatal result in the exceptional instance was due to the advanced age and extremely anæmic and exhausted condition of the petient. She was sixty-one years of age, and had suffered for a very considerable time, and before the operation there was no doubt that the gall-bladder was in a condition of suppuration. She never completely rallied from the operation, and died on the third day. $A$ post-mortem examination was made by Dr. Arthur Foxwell who reported "the heart as being swall, with much pericardial fat; spleen large, soft, and diffluent; advanced cirrhosis of kidneys. The head of the pancreas was the starting point of a fibrous carcinomatous growth, which spread thence to the portal fissure, over which it was scattered in walnut-sized masses. A few pea-sized masses were scattered scantily throughout the liver. I found the drainage tube inserted in an incision one inch long in the base of the gall-bladder on its anterior surface. The gall-bladder was enlarged, empty, and its walls much thickened. About half an inch from the junction of the ducts, the carcinomatous infiltration in the walls of the cystic duct could be detected, but this duct, though constricted, was patent. Both the cystic and hepatic ducts were practically closed very near their junction with the cystic, but not absolutely so. There were one or two foci of suppuration around some bile ducts in the liver. The bile ducts in the liver were generally much dilated, and deeply stained; the whole organ was considerably enlarged, much congested, and in a state of biliary cirrhosis." At the time of the operation the gall-bladder contained a large 